\title{
Centralized Wide Area Damping Controller for Power System Oscillation Problems
}

\author{
Jean Dobrowolski, Petr Korba, Felix Rafael Segundo \\ Sevilla \\ Zurich University of Applied Science \\ ZHAW IEFE, \\ Winterthur, Switzerland \\ dobr@zhaw.ch
}

\author{
Walter Sattinger \\ Swissgrid Ltd \\ Aarau, Switzerland \\ walter.Sattinger@swissgrid.ch
}

\begin{abstract}
In this paper, three different centralized control designs that vary on complexity are presented to damp interarea oscillations in large power systems. All the controls are based on phasor measurements. The first two proposed architectures use simple proportional gains that consider availability of measurements from different areas of the system and fulfill different optimization functions. The third controller is based on a more sophisticated Linear Quadratic Gaussian approach which requires access to the state space model of the system under investigation. The novelty of the proposed scheme resides in designing a single control to command the most influence group of machines in the system. To illustrate the effectiveness of the proposed algorithms, simulations results in the IEEE New England model are presented.
\end{abstract}

Index Terms-Inter-area Oscillation, Particle Swarm Optimization, Linear Quadratic Gaussian, Dynamic Stability, Modal Analysis.

\section{INTRODUCTION}

With the general trend of massive penetration of renewable sources in power systems and the expected decommissioning of nuclear based energy as in Switzerland [1], the future evolution of power grids is a current topic of interest worldwide. Particularly, there is the question if future power systems should evolve in the form of micro-grids, which means to have multi-autonomous small geographical areas where load and generation are integrated locally or continuing developing to even larger super-grids. Each bifurcation has benefits and limitations, for instance micro-grids lack of long distance interconnections having as result absence of inter-area oscillations, however such systems are prone to frequency and other issues if the balance between generation and demand is not meet. On the other hand, super-grids are more robust networks and also significantly more complex to operate. In Europe, the interest for super-grids is evident as demonstrated with the interconnection of Turkey in 2010 to the already complex network of continental Europe [2]. In addition, there is the potential to grow the interconnection to include areas such as the Nordic region or the UK grid, profiting from the existing HVDC connections and building even more [3]. However, despite the robustness and reliability that extra large power systems provide, seldom events like those reported by ENTSO$\mathrm{E}$ in December 2016 on continental Europe, trigger large inter- area oscillation causing severe negative effects on the system [4]. For this reason, damping of inter-area oscillations is a topic of interest within the transmission system operators in Europe, particularly in Switzerland as power system centrally located in the hart of Continental Europe.

Facing inter-area oscillations is not a new topic and most of the solutions proposed can be divided in two directions: improvement of Power System Stabilizer (PSS) by tuning [5], [6] or redesigning [7] its parameters and developing new central schemes such as Wide Area Damping Controllers (WADC) [8], [9] to command generators based on their participation in such events. Designing WADC can vary from simple PID to sophisticate $H_{2} / H_{\infty}$ controllers. In this paper, based on the assumption that readjusting parameters on every existing PSS in a large power grid is ideal but unrealistic, the effects of a new centralized WADC to damp inter-area oscillations is proposed. Three designs that go from a simple proportional gain to a more sophisticated Linear Quadratic Gaussian controller are proposed and explained in detail in section II. The methods for tuning the proposed algorithms and system identification are presented in section III and finally, section IV presents a validation of the methodologies.

\section{Proposed Control Approach}

To deal with the problem of inter-area oscillations, as introduced in Section I, an algorithm based on the idea of adding a centralized WADC to the control loop, as initially suggested in [9], is presented. The proposed WADC receives measurements from Phasor Measurement Units (PMUs) and defines a new input for the excitation system, which is added to the existing controls as PSS. The proposed control sequence of one area, which is used in a multi-area grid is presented by Fig. 1. To illustrate how the proposed approach works, consider first a simple system with only two areas such as the New England IEEE 39 bus system [10], or the widely known Kundur system models [11], respectively. Then, three control variations for the design of a WADC are derived based on different assumptions related to the availability of PMU measurements to feed the WADC system as follows and full details are given in the subsequent subsections:

- Control A $\left(C_{A}\right)$ : Measurements from only one area; 


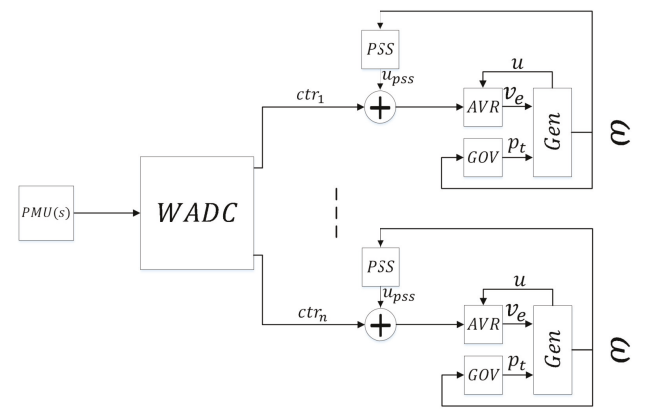

Fig. 1. Control sequence of an area with $n$ Generators (Gen) in a multiple area power systems. The Average Voltage Regulator (AVR) includes the excitation system. The Governor (GOV) is in charge of speed regulation.

- Control B $\left(C_{B}\right)$ : Measurements from both areas;

- Control $\mathrm{C}\left(C_{C}\right)$ : Measurements from both areas and a model of the interactions between the two areas is available.

\section{A. Description of Control A $\left(C_{A}\right)$}

In the first control variation, the PMUs are measuring variables only in the area of interest. In this case, the WADC controller will be an additional control that minimizes the gap between the measured frequency and its reference during an event. To achieve this goal and at the same time deal with the original objective of simplification in power grid control, a simple proportional gain as WADC is used. The control architecture of variation A is presented on Fig. 2. A conven-

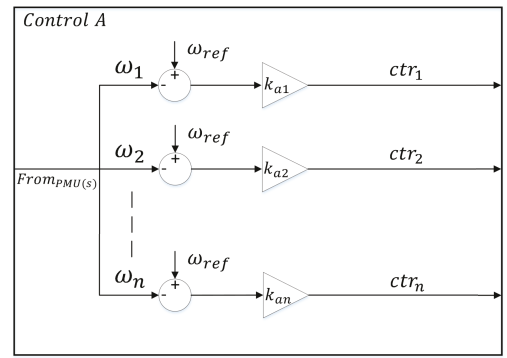

Fig. 2. Design A of WADC. The PMUs are measuring variables of the area of interest and the WADC is an additional control to stabilize frequency (speed).

tional approach for tuning the parameters $k_{a_{i}}, i \in[1, n]$ in the WADC is doing manually, however, this is not straightforward nor simple task for the reason that each generator in the power system under investigation has different participation factor during inter-area oscillations. Thus, more sophisticated algorithms such as the Particle Swarm Optimization (PSO) are available to achieve this task. PSO is a widely used method for power systems regulators tuning [5], [6], [8], [12] and therefore, in this work is used. An introduction and full overview of PSO approach is presented in Section III.

\section{B. Description of Control $B\left(C_{B}\right)$}

In the second design, it is assumed that there are PMU measurements available from both areas. Thus, the control has the capability to minimize the differences between generators by minimizing the frequency variation between the two areas. To deal with the restrictions of $\left(C_{A}\right)$ aforementioned in II-A, a proportional controller depicted on Fig. 3 is proposed. To find the most appropriate value of the parameters $k_{b_{i}}, i \in[1, n]$, PSO approach is also used.

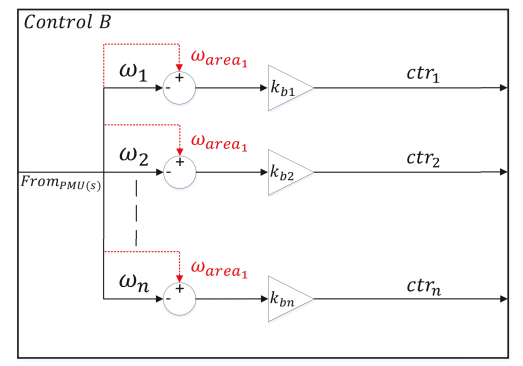

Fig. 3. Design B of WADC. The PMUs are measuring variables in both areas and the WADC is an additional control to minimize the gap between frequencies (speed) of both areas.

\section{Description of Control $C\left(C_{C}\right)$}

In the third control variation, in addition to the assumptions described for $\left(C_{B}\right)$, the following state-space representation of the system is also considered to be available:

$$
\left\{\begin{aligned}
x(k+1) & =A x(k)+B u(k) \\
y(k) & =C x(k)
\end{aligned}\right.
$$

From this representation, a Linear Quadratic Regulator (LQR) controller is proposed based on its flexibility to adapt to the state space system (1). Since this control scheme requires the knowledge of the state vector $x(k)$, which in the case of power systems correspond to generator speeds and angles as well as magnitudes and angles of bus voltages, the association with a Linear Quadratic Estimator (LQE) is required to form the so called Linear Quadratic Gaussian (LQG) control. As demonstrated in [7], the solution of the LQG controller has two components:

- One control sequence $u=-K x$ with $K$ as the solution of the quadratic minimization problem;

- One Kalman gain $G$ such that (2), for $x(k)=\hat{x}(k)$ then $x(k+1)=\hat{x}(k+1)$ else, $\lim _{n \rightarrow+\infty}(x(k+n)-\hat{x}(k+n)) \rightarrow 0$

$$
\begin{cases}\hat{x}(k+1) & =A \hat{x}(k)+B u(k)+\mathbf{G}(y(k)-\hat{y}(k)) \\ \hat{y}(k) & =C \hat{x}(k)\end{cases}
$$

The block diagram of the third design of WADC is depicted in Fig. 4. Subsequent section III introduce the process for identification of matrices $A, B$ and $C$, respectively.

\section{Methodology}

After the three different variations to designs a WADC have been defined, the PSO methodology and its application in power systems to tune controllers is presented. Then, subsection III-C presents the identification of a state-space 


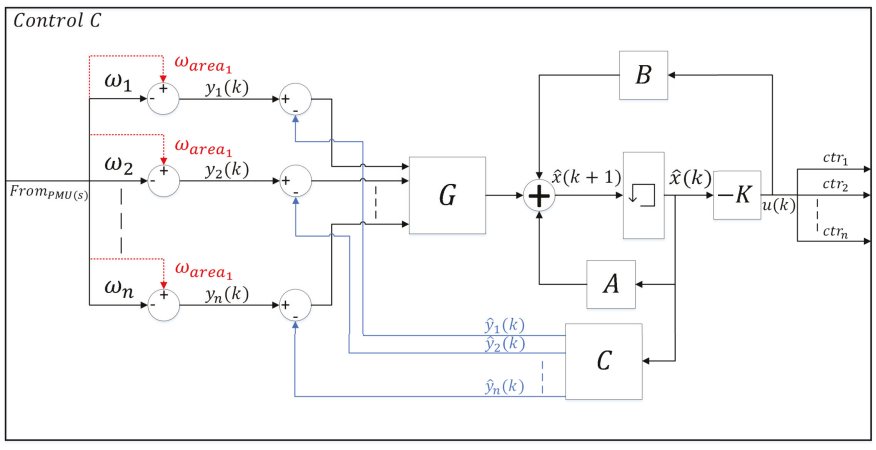

Fig. 4. Design $\mathrm{C}$ of WADC. The PMUs are measuring variables in both areas and a state-space representation of interactions between the two areas is available. The WADC is based on the Linear Quadratic Gaussian theory, $K$ is the regulator gain and $G$ is the Kalman gain to estimate states $x$.

representation of the interactions between two areas in the IEEE New England system. The model identification process is required to calculate the gains $K$ and $G$ introduced on Section II

\section{A. Particle Swarm Optimization theory}

The Particle Swarm Optimization is one of the Swarm method for solving optimization problems. It is an optimization technique, first presented by Eberhat and Kennedy in 1995 [13], inspired by the natural movement of bird and fish groups. The idea of this algorithm is to define a number of particles $n$ where every particle represents a candidate solution for the defined optimization problem $J$, which is represented by an objective function to minimize in this case. Every particle $i$ is characterized by three different factors:

1) Position $P_{i}$ and Velocity $V_{i}$;

2) A measure of quality $J\left(P_{i}\right)$;

3) A best solution since the launch of the algorithm $P_{\text {best }_{i}}$ If all particles are considered, an extra parameter defined as $P_{\text {best }}$ is introduced, which correspond to the global or best possible position that satisfy the inequality $J\left(P_{\text {best }}\right) \leq$ $J\left(P_{\text {best }_{i}}\right) \forall i$ from the launch of the algorithm.

During the first iteration, particles are randomly placed in the searching space restricted by limitations in the position and a its velocity is randomly defined. Then, at each iteration $k$ the position $P_{i}$ and velocity $V_{i}$ of the $i^{t h}$ particle is updated using equation (3):

$$
\left\{\begin{aligned}
V_{i}(k+1) & =H \times V_{i}(k) \\
& +c_{1} \times r \times\left(P_{i}(k)-P_{\text {best }_{i}}\right) \\
& +c_{2} \times r \times\left(P_{i}(k)-P_{\text {best }}\right) \\
P_{i}(k+1) & =P_{i}(k)+V_{i}(k+1)
\end{aligned}\right.
$$

where $H$ is the inertia, $c_{1}$ and $c_{2}$ are weighting coefficients and $r$ is a random number that changes value at each iteration. From (3) is possible to understand that PSO approach operates according to three principles as follows:

1) Momentum, who corresponds to the influence of the current motion related to the inertia $H$;

2) A cognitive part, which corresponds to the attraction to the particle with best solution $P_{\text {best }}$;
3) A social section, related to the amount of attraction to the best position of the tribune (or swarm) $P_{\text {best }}$.

In [5], a good representation of the PSO behavior is proposed as depicted on Fig. 5.

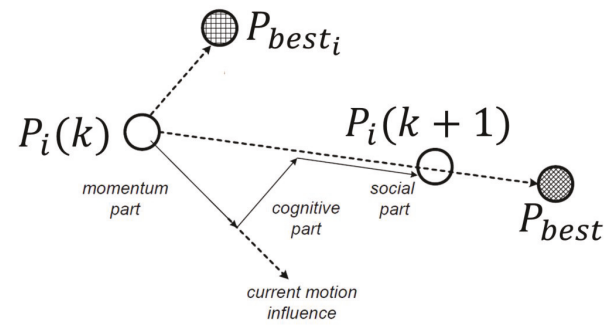

Fig. 5. Representation of Particle Swarm Optimization behavior from [5]. Every particle move following three part: momentum, cognitive and social

Since PSO formulation has some random variables, its performance depends mainly on the number of particles defined. Indeed, the more particles are used, the more space will be cover after the first iteration and the more likely it is to find the global optimum. Nevertheless, is also worth mentioning that the more particles used, the longer will take to the algorithm to converge. One simulation per particle is required at each iteration and thus, more computational time demanded. To ensure the stability of the algorithm, parameters $c_{1}$ and $c_{2}$ must be appropriately tuned to control the velocity of the particles.

\section{B. Particle Swarm Optimization applied in power systems}

PSO has been widely used for power systems controls tuning [?], [5], [6], [8]. Its convergence and stability in case of multidimensional space has been proven in [14]. For this reason, to tune the proposed controllers defined in Sections II-A and II-B, respectively, PSO is applied defining every particle as a $n$ dimensional particle (with $n$ positions) corresponding to the $n$ parameters $k_{(a / b)_{i}}, i \in[1, n]$. In this form, every particle corresponds to a WADC configuration. The particularity of PSO and the quality of its results arises from the definition of the measure of quality $J\left(P_{i}\right)$ introduced on Section III-A.

Considering as a main priority improving the effects of low frequency $(0.1-0.9 \mathrm{~Hz})$ inter-area oscillations in the system [15], two different methods for the definition of optimization function $J$ are considered:

1) First, as proposed in [6], the eigenvalue $\left(\lambda_{i}=\sigma_{i} \pm j \omega\right)$ based multi-objective function defined in equation (4) is considered. Given that improving the damping $(\xi)$ for a particular mode may cause a negative effect on other sensitives modes, the group $\Theta$ denotes the critical modes (within frequency range of interest), which are selected following a simulation without WADC. The final objective is then to drive the selected eigenvalues towards a desired location in the complex plain, represented by shifting the real part $\sigma_{0}$ of the eigenvalue $\lambda_{0}$ and consequently improving its damping ratio $\xi_{0}$.

$$
J_{1}=\sum_{i \in \Theta}\left[\sigma_{0}-\sigma_{i}\right]^{2}+\beta \sum_{i \in \Theta}\left[\xi_{0}-\xi_{i}\right]^{2}
$$


where $\xi_{i}$ the damping ratio of the $i^{\text {th }}$ eigenvalue and $\beta$ a scaling factor.

2) The second method is based on measuring the quality of the actual measurements. Since the proposed controls $C_{A}$ and $C_{B}$ are based on generator speed measurements, the objective function in equation (5) is used to minimize the differences between generators rotor speed $\left(\omega_{r}\right)$ in the two areas while maintaining the regulation to the nominal value $\omega_{\text {ref }}$ and minimizing the number of oscillations $N_{\text {osc }}$.

$$
J_{2}=\sum_{i \in a_{2}}\left[\omega_{a_{1}}-\omega_{i}\right]^{2}+\sum_{i \in a_{2}}\left[\omega_{i}-\omega_{r e f}\right]^{2}+\beta N_{o s c}
$$

where $\omega_{a_{1}}$ is the average speed in area one and $\beta$ a scaling factor.

\section{Identification of inter-area interactions}

In this section, identification of the state-space representation of the interaction of the inter-area oscillations is proposed. In our previous work [7] it has been proved that accurate dynamic identification of the system allows to design an adaptive control and then to improve the damping of interarea oscillations. Due to the fact that in this work power system simulations have been performed using the commercial software DigSilent PowerFactory, the proposed adaptive control requires co-simulation with Matlab, which has been proved to be computational expensive, specially for large power systems. Considering that the dynamic identification approach presented in [7], which is referred as Eigensystem Realization Algorithm (ERA), has the drawback of relying on an impulse as control input. For this reason, in this work a more conventional identification method to design the third control architecture introduced on Section II-C was selected. In order to find an good representation of the inter-area oscillation interactions, one possibility is to consider the state-space (1) with $y \in \Re^{n}$ corresponding to variations between generator rotor speeds of the $n$ generators in area 2 with the average speed of area 1 and $u \in \Re^{n}$ as the PSS output, corresponding to $u_{p s s}$ in Fig. 1. To achieve this goal, it is possible to use statespace identification subroutines such as the MATLAB/N4SID.

The IEEE-39 bus system depicted on Fig. 6 has 5 inter-area modes in the range of $[0.59-0.97] \mathrm{Hz}$. In this work, special attention is placed to the mode with smallest frequency since it is the weakest and represents the interaction between G1 (Area 1) gainst rest of the generators (area 2). In this system, the following event was simulated: A $29 \mathrm{sec}$ simulation was performed, where at $1 \mathrm{sec}$ the line 16 was suddenly tripped and the WADC controller was disable. After performing the simulation, the frequency of G1 was compared against the frequency of the nine remaining machines and the vector $y(k)$ in (1) was formed. In the commercial software PowerFactory, is also possible to have access to the PSSs output and build the control vector $u(k)$. Since there is no information a priory about the actual order of the system, a model reduction process is performed and the results are depicted on Fig. 7(a). From this subplot, it can be observed that a new system of order 20 provides the lowest error between the reduced model and the original system. Figs. 7-(b-j) are a comparison between the simulation of the estimated state-space and the real measurement in the rest of the generators.

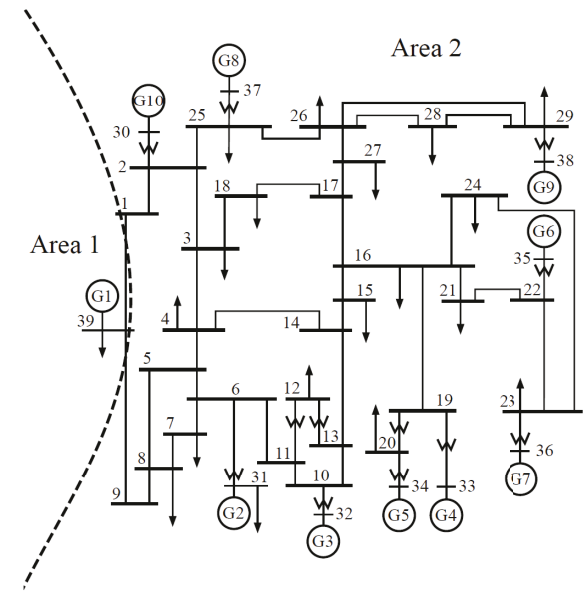

Fig. 6. Representation of Particle Swarm Optimization behavior from [5]. Every particle move following three part: momentum, cognitive and social

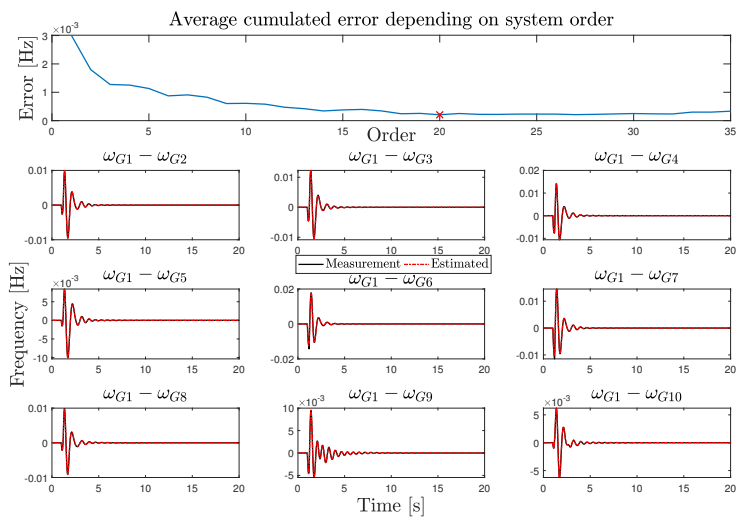

Fig. 7. Representation of Particle Swarm Optimization behavior from [5]. Every particle move following three part: momentum, cognitive and social

\section{Power Systems Application \& Main Results}

To demonstrate the effectiveness of the proposed control schemes presented on Section II, the IEEE New England system depicted on Fig. 6 has been used. The power system has been subject to a randomly selected 3-phase short-circuit at line 16 after $1 \mathrm{sec}$ of simulation and the fault was cleared after $180 \mathrm{msec}$. Note that the proposed controls $C_{A}, C_{B}$, and $C_{C}$ have different methodology and thus, the software configuration depends of the control to be applied.

\section{A. Performance Evaluation of Controls $C_{A} \& C_{B}$}

To find the optimal parameters for controls $C_{A}$ and $C_{B}$, respectively, PSO method was applied. To integrate the control objectives into the DigSilent PowerFacrtory environment, the Application Programming Interface (API) that link python 
with the power system software was operated. The principle is to launch a python code that controls parameters within PowerFactory whenever is requited, and at the same time to have access into the stored results. Hence, a subroutine for a application of PSO with $p$ number of particles for $i$ iterations was developed and the flow chart of the subroutine is depicted on Fig. 8. In this figure, blue instructions represent special actions for the evaluation of the optimization function $J_{1}$, while red instructions represent special actions for the evaluation of the optimization function $J_{2}$ and black instructions are common actions for both optimization functions .

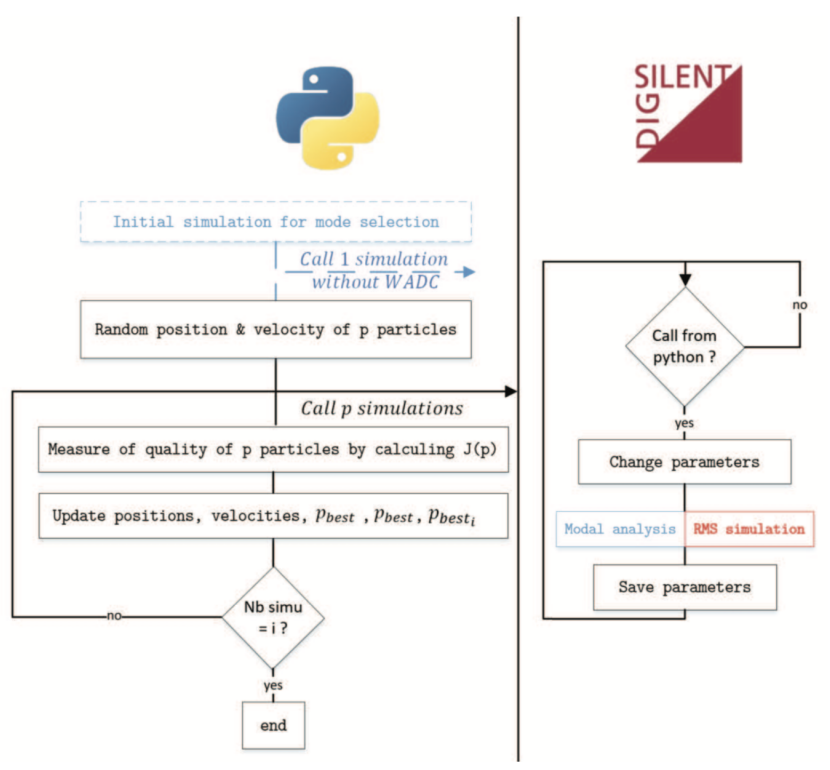

Fig. 8. Subroutine to apply PSO with python and PowerFactory. The blue actions are specials for $J_{1}$ evaluation, the red ones for $J_{2}$ and the black one are common actions.

From 250 tests, the optimal configuration for tuning of variables in equation (3) was found and the results are summarized on table I.

TABLE I

PARTICLE SWARM OPTIMIZATION BEST CONFIGURATION

\begin{tabular}{|c|c|c|c|c|c|}
\hline $\begin{array}{c}\text { Inertia } \\
H\end{array}$ & $c_{1}$ & $c_{2}$ & $\begin{array}{c}\text { Number of } \\
\text { Particles }\end{array}$ & $\begin{array}{c}\text { Number of } \\
\text { iteration }\end{array}$ & $\begin{array}{c}\text { Range of } \\
k_{(a / b)_{i}}\end{array}$ \\
\hline 0.3 & 0.9 & 1.1 & 100 & 70 & {$[0.01-100]$} \\
\hline
\end{tabular}

Using this configuration, the tuning of the proposed controls $C_{A}$ and $C_{B}$ for implementation of the objective functions $J_{1}$ and $J_{2}$, was carried on and the final results are depicted on Table II. For comparisons purposes, the value of the objective functions without WADC are $J_{1}=550.651$ and $J_{2}=62154.2$, respectively.

\section{B. Performance Evaluation of Controls $C_{C}$}

The control $C_{C}$ has fewer constraints than controllers $C_{A}$ and $C_{B}$ and therefore no co-simulations are required, which
TABLE II

TUNING OF CONTROLS $C_{A}$ AND $C_{B}$ FOR DIFFERENT OBJECTIVE FUNCTIONS (OF)

\begin{tabular}{|c|c|c|c|c|}
\hline $\begin{array}{c}k_{(a / b)_{i}} \\
\text { OF- } J_{i}\end{array}$ & $\begin{array}{c}C_{A} \\
\text { OF- } J_{1}\end{array}$ & $\begin{array}{c}C_{B} \\
\text { OF- } J_{1}\end{array}$ & $\begin{array}{c}C_{A} \\
\text { OF- } J_{2}\end{array}$ & $\begin{array}{c}C_{B} \\
\text { OF- } J_{2}\end{array}$ \\
\hline$k_{(a / b)_{1}}$ & 0.01 & 94.600 & 68.804 & 91.231 \\
\hline$k_{(a / b)_{2}}$ & 20.768 & 25.736 & 37.954 & 29.245 \\
\hline$k_{(a / b)_{3}}$ & 39.475 & 97.177 & 20.969 & 96.021 \\
\hline$k_{(a / b)_{4}}$ & 93.687 & 58.6967 & 23.569 & 49.568 \\
\hline$k_{(a / b)_{5}}$ & 49.713 & 6.841 & 57.285 & 10.254 \\
\hline$k_{(a / b)_{6}}$ & 79.819 & 85.303 & 1.116 & 82.187 \\
\hline$k_{(a / b)_{7}}$ & 26.635 & 92.851 & 0.090 & 96.329 \\
\hline$k_{(a / b)_{8}}$ & 78.989 & 11.678 & 0.01 & 9.548 \\
\hline$k_{(a / b)_{9}}$ & 87.928 & 72.948 & 11.857 & 68.245 \\
\hline OF & 516.885 & 274.804 & 50857.4 & 40533.6 \\
\hline
\end{tabular}

speed-up simulation time and hence only PowerFactory is required. The main challenge related to this controller is a limitation from the power system software related to how matrix calculations are handled. To overcome this issue, the reduced order model of the IEEE New England system of 20 states was built from scratch as a new DSL model within DigSilent Power Factory [16].

\section{Main Results}

In this section, the performance of the proposed controllers are presented for different optimization functions $\left(O F-J_{i}\right)$, as presented on Table II. In total, five possible combinations using the three different controllers were investigated:

- Case study 1: $C_{A}$ for $O F-J_{1}$;

- Case study 2: $C_{A}$ for $O F-J_{2}$;

- Case study 3: $C_{B}$ for $O F-J_{1}$;

- Case study 4: $C_{B}$ for $O F-J_{2}$;

- $\overline{\text { Case study 5: }} C_{C}$.

To compare the performance of the proposed controllers under different case studies, generators speed measurements from both areas in the IEEE New England system during RMS simulations are presented. For sake of simplicity, Fig. 9 (a)(d) compares the speed variation of one generator from each area: G1 vs G6 (randomly selected) following a 3-phase fault of $180 \mathrm{msec}$ applied to line 16 as in the previous case. Fig. 9 (a) depicts the simulation result without WADC. Fig. 9 (b) presents the results of the case studies 1 and 3. Fig. 9 (c) show the simulation results for case studies 2 and 4 and finally, Fig. 9 (d) depicts the simulation results for case study 5 , which is the $C_{C}$ LQG controller. From the results presented on Fig. 9, the following remarks are derived:

- When no control is used, the inter-area mode with a frequency of $0.59 \mathrm{~Hz}$ is clearly visible in the response of the generators, which generates active power fluctuation in the order of $500 \mathrm{MW}$ that can severely affect the objectives defined in the optimizing problem.

- From subplot 9 (b) it can be observed that $C_{A}$ minimize the amplitude of the oscillations, but do not improve the stability of the inter-area mode in G1. The measure of $J_{1}$ for $C_{A}$ in Table II confirm this, with no significant 

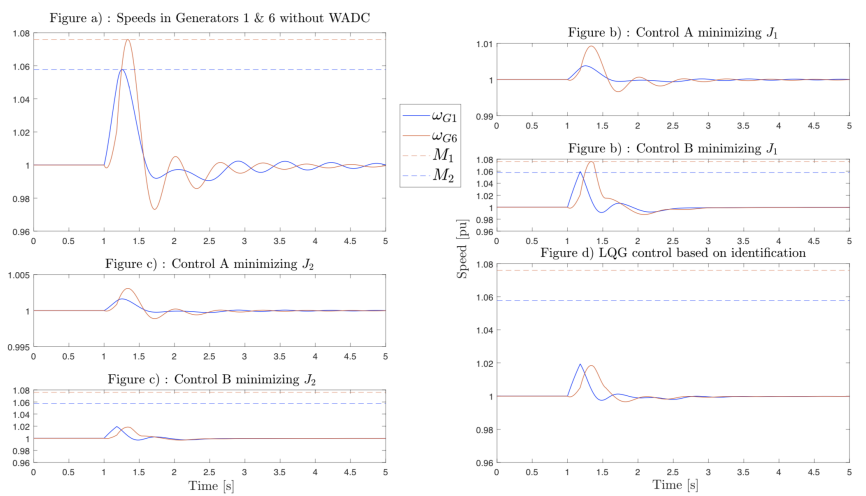

Fig. 9. (a) No control, (b) $C_{A}$ and $C_{B}$ to minimize $O F-J_{1}$, (c) $C_{A}$ and $C_{B}$ to minimize $O F-J_{2}$ and (d) $\mathrm{LQG}$ control.

improvement compared to the case without WADC. On the other hand, $C_{B}$ presents significant improvement, damping the oscillation fast (approximately 2.5 seconds after the fault). Since $J_{1}$ is designed only to improve the stability of selected eigenvalues, $C_{B}$ has no internal characteristics to automatically reduce the amplitude of the oscillations and hence the final amplitudes are not significantly reduced for this example.

- From subplot 9 (c), it can be seen that the results are similar to subplot 9 (b). The main difference is that, the objective function $J_{2}$ described on equation (5), includes a component to minimize the amplitude. For this reason $C_{A}$ and $C_{B}$ provide better performance than in the previous case. Another remark is that the first element of $J_{2}$, is used to minimize the difference between generator speeds in both areas and based in the simulation results, it can be concluded that this effect is as efficient as the eigenvalue based multi-objective function.

- Subplot 9 (d) demonstrates the effectivenes of $C_{C}$ for case study 5 . The controller reduce the amplitude of the oscillations and provide a significant damping in about 3 seconds.

The proposed controllers present different behavior from which $C_{A}$ provides the weakest performance to damp interarea oscillations. $C_{A}$ can positively influence the amplitudes of the oscillations, but cannot improve the stability of the system, which is the main objective in this work. On the other hand, $C_{B}$ and $C_{C}$ are the most efficient control schemes in terms of minimize oscillation amplitude while significantly improving the damping of the system, the challenge to implement the proposed architectures is that they require global measurement and a global communication with PMUs, which can be prone to communication problems.

\section{CONCLUSIONS}

Three different designs for WADC to damp inter-area oscillations have been presented. The first two architectures are based on proportional gains that consider availability of PMU measurements from different areas of the system and fulfill different optimization functions. The third controller is based on a more sophisticated Linear Quadratic Gaussian approach, which requires access to the state space model of the system under investigation. Performance of control $C_{A}$ indicates that measurements from one area are not sufficient to damp interarea oscillations. Similarly, simulation results based on $C_{B}$ and $C_{C}$ demonstrate that with measurements from more than one area is possible to significantly improve damping of interare modes. Moreover, from the results obtained with control $C_{B}$ based on $O F-J_{1}$ and $O F-J_{2}$ it can be noticed that is possible to enhance stability of the system as good as with approaches based on modal analysis.

\section{ACKNOWLEDGMENT}

The authors acknowledge the Swiss National Science Foundation (SNSF) under the program Ambizione Energy Grant (AEG). This research is also part of the activities of SCCER FURIES, which is financially supported by the Swiss Innovation Agency (Innosuisse-SCCER program)

\section{REFERENCES}

[1] Energy Strategy 2050, Swiss Federal Office of Energy (SFOE), 2017. [Online]. Available: http://www.bfe.admin.ch/energiestrategie2050/index.html?lang=en

[2] I. A. Nassar and H. Weber, "System analysis of the Turkish power system for interconnection with continental Europe," IFAC Proceedings Volumes, vol. 45, no. 21, pp. 168 - 173, 2012.

[3] Trans-European Infrastructure - Projects of Common Interest, European Commission, 2014. [Online]. Available: https://ec.europa.eu/inea/sites/inea/files/download/publications /pcienersuperfinal.pdf

[4] Analysis of ce inter-area oscillations of 1st december 2016, entsoe, 2016, available at https://docstore.entsoe.eu/Documents/SOC20documents/Regional GroupsContinentalEurope/2017/CEinterareaoscillationsDec1st2016PUBLICV7.pdf.

[5] S. Panda and N. P. Padhy, "Coordinated design of tcsc controller and pss employing particle swarm optimization technique," vol. 1, no. 4, pp. 706-714, 2007.

[6] A. Stativa, M. Gavrilas, and V. Stahie, "Optimal tuning and placement of power system stabilizer using particle swarm optimization algorithm," in Electrical and Power Engineering (EPE), 2012.

[7] J. Dobrowolski, F. R. Segundo, F. A. Zelaya, and M. R. A. Paternina, "Inter-area Oscillation Control Based on Eigensystem Realization Approach," in 2018 IEEE Autumn Meeting on Power, Electronics and Computing (ROPEC 2018), Ixtapa, Mexico, Nov. 2018. [Online]. Available: https://hal-iogs.archives-ouvertes.fr/hal-01926442

[8] M. Zamani, N. Sadati, and M. K. Ghartemani, "Design of an ho pid controller using particle swarm optimization," International Journal of Control, Automation and Systems, vol. 7, no. 2, pp. 273-280, 2009.

[9] Y. Zhang and A. Bose, "Design of wide-area damping controllers for inter-area oscillations," IEEE Transactions on Power Systems, Aug 2008.

[10] S. Dutta and S. P. Singh, "Optimal rescheduling of generators for congestion management based on particle swarm optimization," IEEE Transactions on Power Systems, 2008.

[11] M. Klein, G. J. Rogers, and P. Kundur, "A fundamental study of inter-area oscillations in power systems," IEEE Transactions on Power Systems, vol. 6, no. 3, pp. 914-921, Aug 1991.

[12] TZ.-L. Gaing, "A particle swarm optimization approach for optimum design of pid controller in avr system," IEEE transactions on energy conversion, vol. 19, no. 2, pp. 384-391, 2004.

[13] J. Kennedy, "Particle swarm optimization," pp. 760-766, 2011.

[14] M. Clerc and J. Kennedy, "The particle swarm-explosion, stability, and convergence in a multidimensional complex space," IEEE transactions on Evolutionary Computation, vol. 6, no. 1, pp. 58-73, 2002.

[15] P. Kundur, N. J. Balu, and M. G. Lauby, Power system stability and control. McGraw-hill New York, 1994, vol. 7.

[16] DIgSILENT, Power Factory Users Manual, DIgSILENT GmbH, 2018. 